\title{
FREQUENCY OF ERG-POSITIVE PROSTATE CARCINOMA in Poland
}

\author{
Karolina KaczmarczyK ${ }^{1}$, Grzegorz Dyduch ${ }^{1}$, Magdalena Bialas ${ }^{1}$, Sergiusz DemczuK ${ }^{1}$, \\ Tomasz Szopiński ${ }^{2}$, Piotr ChŁosta ${ }^{2}$, KrzyszTof OKOŃ $^{1}$
}

${ }^{1}$ Department of Pathomorphology, Jagiellonian University, Krakow, Poland

2Department of Urology, Jagiellonian University, Krakow, Poland

\begin{abstract}
Prostatic carcinoma (PC) is one of the most frequent cancers in men. Molecular pathogenesis of PC remains poorly understood. Translocations involving ERG were found to be the single most frequent genetic event. A strong correlation exists between this translocation and ERG positivity on immunohistochemistry. The rate of ERG positivity and its relationship with other clinicopathological parameters differ between populations and between studies; in particular, there are few data on ERG-positive PC in Eastern Europeans. In the present study, tissue microarrays of unselected PC cases were constructed and standard immunohistochemistry for ERG performed. The results were compared with the basic pathologic prognostic parameters. The group under study consisted of 113 cases; $52(46.02 \%)$ were positive for ERG. The positive cases showed a slightly higher Gleason score (median 6 vs. 7). The majority of ERG-positive cases showed nerve bundle invasion and were also less likely to be prostate confined than negative ones. In conclusion, the frequency of ERG-positive PC in our series is similar to Western populations, and they show some unfavorable prognostic features.
\end{abstract}

Key words: prostatic carcinoma, ERG, tissue microarray, immunohistochemistry.

\section{Introduction}

Prostatic carcinoma (PC) is one of the most frequent cancers in males in the Western world [1]; indeed it is first in incidence in the North American population, and second in frequency in Poland. The molecular basis of PC remains poorly understood, and is the subject of intense study. While most human carcinomas show complex genetic changes and recurrent translocation is seen more often in leukemia or sarcomas, in 2005 it was shown that a significant subset of PCs bear translocations involving members of the erythroblast transformation-specific (ETS) gene family, usually v-ets avian erythroblastosis virus E26 oncogene homolog (ERG) [2]. Later it was shown that ERG translocation is strongly correlated with ERG-protein expression as detected by immunohistochemistry [3-5], opening the possibility of easy studies.

The frequency of ERG translocation, its relationship to stage, grade and prognosis, and its diagnostic utili- ty remain a matter of debate. Interestingly, these relationships were suggested to differ between ethnic groups; in particular ERG-positive PC is frequent in North American and Western European populations, but much less so in Asians $[6,7]$. The differences in molecular biology of PC may depend on purely genetic factors, but also on frequency of occurrence, age structure of the population and differences in detection strategies. Also, the search for prognostic factors is important in $\mathrm{PC}$ for distinguishing aggressive from indolent ones. One of the potential biomarkers is angiogenesis [8], and ERG expression could be another one. Although it may be surmised that the PCs in Central-Eastern Europe are similar to the cases from the Western population, hard evidence is needed to support this. As no data are available on ERG positivity in Polish prostatic carcinoma patients, we decided to perform a tissue microarray study.

Preliminary analysis of a subset of the data was presented in poster form at the $25^{\text {th }}$ European Congress 
of Pathology, held in Lisbon, Portugal (31 August to 4 September 2013).

\section{Material and methods}

The material for the study consisted of unselected prostatectomy specimens from the files of the Pathology Department. The slides were reviewed by an urologic pathologist. The cases were reclassified according to the current Gleason system and staged according to current TNM criteria [9, 10]. Nerve invasion, lymphovascular invasion, status of surgical margins, presence of multiple tumor foci, and production of mucin were also reevaluated. From each case one representative section was chosen. On the slide, the region of interest containing carcinoma tissue was marked, then its extent was copied to the surface of the paraffin block. For the tissue microarray (TMA) production a manual device (Histopathology Inc., Hungary) was used. From the region marked as cancer on each paraffin block, two $2 \mathrm{~mm}$ cores were obtained and transferred into a recipient block. The case numbers with respective location in the TMA were noted on an Excel (Microsoft Inc., USA) spreadsheet. The upper-left corner of the TMA was left empty to allow proper orientation of the resulting slides. From the TMA paraffin blocks, $2 \mu \mathrm{m}$ sections were prepared and stained with hematoxylin-eosin
(HE) and immunohistochemistry. HE slides were used to control the quality of tissue selection and determine the Gleason score of specific spots. Immunohistochemistry was done in the routine manner. A rabbit monoclonal anti-ERG antibody (clone EPR3864), produced by Abcam, was used in $1: 200$ dilution. Heating in citrate buffer for 30 minutes was used for antigen retrieval. The LabVision detection system (Thermo Scientific, USA) was used. The results were scored as positive when unequivocal nuclear staining was present; very faint nuclear and any cytoplasmic reaction were ignored (Fig. 1). The results were collected in an Excel spreadsheet containing the case numbers. Statistics were calculated with Statistica 10 (StatSoft Inc., USA). Mann-Whitney $\mathrm{U}$ and $\chi^{2}$ tests were used, as appropriate. The significance level was set to 0.05 .

\section{Results}

Analyzed material consisted of 113 cases. The mean age of the patients was 62.09 years (range 42 to 78 , SD 6.53). Stage of the tumors was pT2a in 7 cases $(6.19 \%)$; p 2 b in 3 cases $(2.65 \%)$; pT2c in 34 cases $(30.09 \%)$; p 3 a in 51 cases $(45.13 \%)$; $\mathrm{pT} 3 \mathrm{~b}$ in 16 cases $(14.16 \%)$; p 44 in 2 cases $(1.77 \%)$. In 98 cases (86.73\%) lymph nodes contained no metastatic deposits; in 2 cases $(1.77 \%)$ there were lymph node metas-
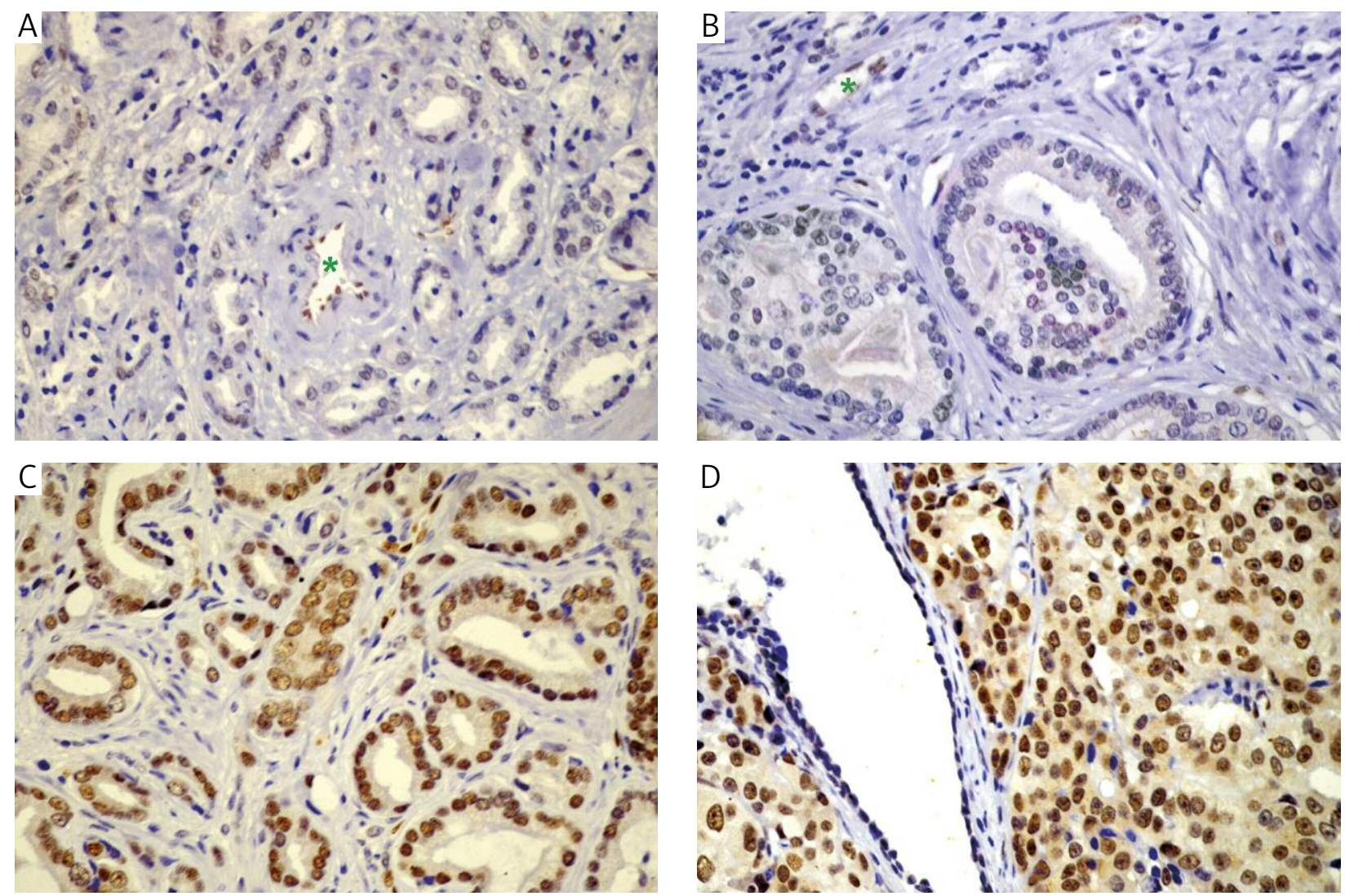

Fig. 1. Immunohistochemistry for ERG in prostatic carcinoma. A, B negative cases (notice the positive reaction in endothelia (*), C, D positive cases (notice lack of stain in non-neoplastic and stromal cells). A, C Gleason pattern 3, B, D Gleason pattern 4 . Original magnification $400 \times$ 
tases, while in 13 cases $(11.50 \%)$ no lymph nodes were sampled. In 62 cases $(54.87 \%)$ the surgical margins were negative, while in 51 cases $(45.13 \%)$ positive surgical margins were found.

Gleason score was 6 in 46 cases $(40.71 \%) ; 7$ in 54 cases $(47.79 \%) ; 8$ in 6 cases $(5.31 \%) ; 9$ in 7 cases $(6.19 \%)$. The frequency of individual Gleason patterns is shown in Table I. Tertiary Gleason pattern was seen in 18 cases, it was pattern 3 in 2 cases $(1.77 \%)$, pattern 4 in 11 cases $(9.73 \%)$, and pattern 5 in 5 cases $(4.42 \%)$. Appreciable mucin production was seen in 9 cases $(7.96 \%)$. Multifocality was seen in 16 cases (14.16\%). Lymphovascular invasion was seen in 39 cases $(34.51 \%)$ and infiltration of nerve bundles in 69 cases $(61.06 \%)$. In 3 cases $(2.66 \%)$ intraductal carcinoma was present.

A positive reaction for ERG was present in 52 cases (46.02\%). The patients with ERG-positive PC were slightly younger (61.69 years, SD 7.04 vs. 62.43 years, SD 6.09) but this difference was not statistically significant. The positive cases showed a higher Gleason score (median 7 vs. $6, \mathrm{p}<0.05$ ). To further explore the relationship between Gleason grade and ERG expression, the pattern seen in individual cores was noted and compared between immunopositive and negative cases. Among the ERG-positive cases the proportion of pattern 4 was significantly higher than among negative cases ( $41.11 \%$ vs. $23.28 \%, \mathrm{p}<0.022$; Fig. 2). ERG-positive cases were less likely to be prostate confined ( 15 out of 52 vs. 29 out of 61 for negative cases, $\mathrm{p}<0.043)$. There were no significant differences when considering the $\mathrm{pT}$ subcategories, however. The majority of ERG-positive cases showed nerve bundle invasion ( 38 out of 52 vs. 31 out of 61 for negative cases, $p<0.016$ ). There was no difference between ERG-positive and negative cases in other analyzed parameters.

\section{Discussion}

In ERG-positive PC, the translocation moves the ERG coding sequence (or less frequently another ETS
Table I. Details of Gleason grades

\begin{tabular}{lcc}
\hline GLEASON PATTERNS AND SCORES & $\mathbf{N}$ & $\%$ \\
\hline $3+3=6$ & 46 & 40.71 \\
\hline $3+4=7$ & 41 & 36.28 \\
\hline $3+5=8$ & 2 & 1.77 \\
\hline $4+3=7$ & 13 & 11.50 \\
\hline $4+4=8$ & 3 & 2.65 \\
\hline $4+5=9$ & 4 & 3.54 \\
\hline $5+3=8$ & 1 & 0.88 \\
\hline $5+4=9$ & 3 & 2.65 \\
\hline
\end{tabular}

gene family member) in the region controlled by the androgen receptor regulatory sequence (predominantly the TMPRSS2 gene). This results in ERG mRNA and protein overexpression. The overexpressed ERG protein promotes cancer development and progression. This appears to be an early event in prostatic carcinogenesis as, at least in some studies, ERG alterations are seen in prostatic intraepithelial neoplasia (PIN), but not in its putative precursor, proliferative inflammatory atrophy $[2,11,12]$.

To the best of our knowledge, no study concerning ERG-positive prostatic carcinoma in the Polish population has been published so far. In fact, there is little information concerning the Central Eastern European population. Although basically people originating from this region are not dissimilar from Western Europeans, some genetic differences may exist. Beside the genetic differences, the PC in our country has a shorter history of PSA screening and there is a smaller population of elderly persons. All these might influence the frequency of ERG positivity of PC.

The prevalence of PC related to ERG alterations is well studied in Western-European and North-American populations, although its relationship with prognosis remains controversial. Furusato et al. reported $45 \%$ of ERG-positive PCs [11]; they observed a strong correlation between ERG expression in carcinoma and ad-

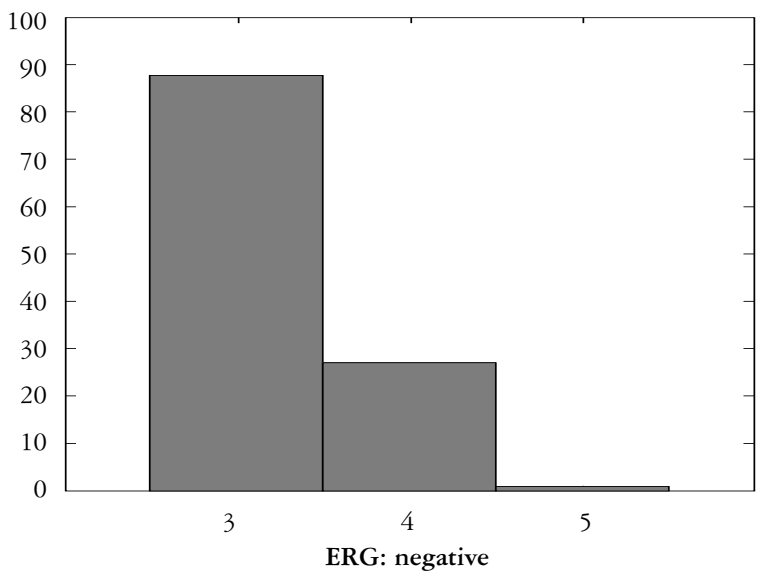

Fig. 2. Frequency of individual Gleason patterns in ERG-positive and ERG-negative TMA cores 
jacent PIN foci. ERG-positive cancers tended to show higher grades. Van Leenders et al. [5] in biopsy material estimated the rate of ERG positivity as $61 \%$ of cases and positive tumors tended to be larger than negative ones. In the same study $73 \%$ of prostatectomy specimens were positive; no relationship with stage or grade was visible, yet the analyzed group was small. Also in the preliminary analysis of the present study, and with similar sample size to that of the van Leenders et al. study, we were not able to detect a relationship between ERG and grade, which became evident when the study group expanded. Verdu et al. [13] found that $49 \%$ of Spanish PCs show ERG positivity. In their material no relationship with Gleason grade or stage was present. Also, they failed to see ERG expression in PIN. Szasz et al. in a small study [14] found a positive correlation between ERG expression and Gleason grade; they also observed more ERG-positive cases in advanced cancers than in prostate-confined ones. These results are very similar to the relationships seen in our material. Teng et al. estimated the frequency of ERG-positive PC as $46 \%$ [15]. Remarkably, the frequency of ERG positivity was significantly lower in locally advanced as well as metastatic PCs. Fine et al. [16] found $41 \%$ of TMPRSS2ERG translocation and an additional 9\% of amplification of the same region. Cases with translocation showed a lower Gleason grade, while cases with amplification showed a higher one. Fine's study did not analyze the stage of the tumors. Albadine et al. $\{17\} \mathrm{lim}-$ ited their analysis to low Gleason grade $(<7)$, prostate confined tumors with a volume $<0.5 \mathrm{~cm}^{3}$, and compared them to more advanced yet still low-grade carcinomas. The authors observed slightly lower frequency of TMPRSS2-ERG fusion in minute carcinomas $(47 \%$ vs. $62 \%$ ) yet the difference was not statistically significant. Also, in contrast to the present study, the frequency of the ERG alteration was slightly lower in pT3 than in pT2 cases. Most importantly, Albadine et al. did not find an association between ERG status and the risk of biochemical recurrence [17].

The prevalence of ERG translocation in PC of Asiatic patients is significantly lower. In the Korean population, Lee et al. [7] found that only 20\% of PCs were ERGtranslocation dependent, and in similar material Suh et al. estimated this frequency as $24 \%$ [18]. What is more, in both studies, ERG-positive cases tended to be lower grade (Gleason score $\leq 7$ ) and no relationship with biochemical recurrence rate, age, stage or invasiveness was observed. In particular, cancers with large cribriform glands (typically pattern 4) were unlikely to express ERG. In the Japanese, Kimura et al. [19] found only 16\% ERG positivity. These cases tended to be younger, lower grade and margin-negative. Mao et al. [6] compared the genetic features of $\mathrm{PC}$ in Chinese and British populations. They found that in Chinese ERG translocation is quite rare $(8 \%)$. They detected no relationship between age or Gleason grade and ERG status.
To explain the inconsistency of the relationship between ERG expression and prognosis Wu et al. [20] hypothesized that the translocation seen in various cancers may be in fact different. In some cases the ERG partner of the fusion gene might be truncated, leading to production of a protein product with no function or even an inhibitory protein binding to ETS domain binding sites and blocking them. This hypothesis correlates with the different significance of translocation and amplification of the ERG region [16]. In a recent paper, Tandefelt et al. [21] were able to define two distinct groups of ERG-positive PC according to gene expression which resulted in different clinical outcomes, while ERGnegative cases formed another cluster of cases. On the other hand, in their study, ERG status alone was not predictive of the patients' outcome. As pointed out by Fine et al. [16] some problems in reporting the significance of ERG expression for prognosis may also depend on inconsistency in the way Gleason grade is reported. For this reason in the present work the grading was repeated and done by the current ISUP recommendation.

Some authors have proposed diagnostic and prognostic use of ERG immunohistochemistry [5, 22-25]. The rationale for such use is very high specificity of the translocation, its appearance early in the course of disease and persistence in the course of cancer progression. In fact, ERG positivity was not described in cases of prostatic cancer mimickers. On the other hand, ERG positivity is obviously a low-sensitivity test. In our opinion, for the time being, extreme caution is needed in diagnostic use of ERG immunohistochemistry. Also, the prevalence of ERG positivity in PC with extraprostatic extension and higher grade might make it useful for distinguishing aggressive and indolent carcinomas. Unfortunately, the relationship seen in this and a few other studies is not confirmed by other authors.

In conclusion, we have shown for the first time that prostatic carcinoma in Polish patients shows a similar rate of ERG expression to patients of Western European descent. Our ERG-positive cases also tend to display unfavorable prognostic factors such as higher stage and grade.

The authors declare no conflict of interest.

\section{References}

1. Jemal A, Bray F, Center MM, et al. Global cancer statistics. CA Cancer J Clin 2011; 61: 69-90.

2. Tomlins SA, Rhodes DR, Perner S, et al. Recurrent fusion of TMPRSS2 and ETS transcription factor genes in prostate cancer. Science 2005; 310: 644-648

3. Park K, Tomlins SA, Mudaliar KM, et al. Antibody-Based Detection of ERG Rearrangement-Positive Prostate Cancer. Neoplasia 2010; 12: U590-U595.

4. Falzarano SM, Zhou M, Carver P, et al. ERG gene rearrangement status in prostate cancer detected by immunohistochemistry. Virchows Arch 2011; 459: 441-447. 
5. van Leenders GJ, Boormans JL, Vissers CJ, et al. Antibody EPR3864 is specific for ERG genomic fusions in prostate cancer: implications for pathological practice. Mod Pathol 2011; 24: 1128-1138.

6. Mao X, Yu Y, Boyd LK, et al. Distinct genomic alterations in prostate cancers in Chinese and Western populations suggest alternative pathways of prostate carcinogenesis. Cancer Res 2010; 70: 5207-5212.

7. Lee K, Chae JY, Kwak C, et al. TMPRSS2-ERG gene fusion and clinicopathologic characteristics of Korean prostate cancer patients. Urology 2010; 76: 1268.e1267-1213.

8. Luczynska E, Gasinska A, Wilk W. Microvessel density and expression of vascular endothelial growth factor in clinically localized prostate cancer. Pol J Pathol 2013; 64: 33-38.

9. Epstein JI, Allsbrook WC Jr., Amin MB, et al. The 2005 International Society of Urological Pathology (ISUP) Consensus Conference on Gleason Grading of Prostatic Carcinoma. Am J Surg Pathol 2005; 29: 1228-1242.

10. Fine SW, Amin MB, Berney DM, et al. A contemporary update on pathology reporting for prostate cancer: biopsy and radical prostatectomy specimens. Eur Urol 2012; 62: 20-39.

11. Furusato B, Tan SH, Young D, et al. ERG oncoprotein expression in prostate cancer: clonal progression of ERG-positive tumor cells and potential for ERG-based stratification. Prostate Cancer Prostatic Dis 2010; 13: 228-237.

12. Perner S, Mosquera JM, Demichelis F, et al. TMPRSS2-ERG fusion prostate cancer: an early molecular event associated with invasion. Am J Surg Pathol 2007; 31: 882-888.

13. Verdu M, Trias I, Roman R, et al. ERG expression and prostatic adenocarcinoma. Virchows Arch 2013; 462: 639-644.

14. Szász AM, Majoros A, Rosen P, et al. Prognostic potential of ERG (ETS-related gene) expression in prostatic adenocarcinoma. Int Urol Nephrol 2013; 45: 727-733.

15. Teng LH, Wang C, Begin LR, et al. ERG protein expression and gene rearrangements are present at lower rates in metastatic and locally advanced castration-resistant prostate cancer compared to localized disease. Urology 2013; 82: 394-399.

16. Fine SW, Gopalan A, Leversha MA, et al. TMPRSS2-ERG gene fusion is associated with low Gleason scores and not with highgrade morphological features. Mod Pathol 2010; 23: 1325-1333.

17. Albadine R, Latour M, Toubaji A, et al. TMPRSS2-ERG gene fusion status in minute (minimal) prostatic adenocarcinoma. Mod Pathol 2009; 22: 1415-1422.

18. Suh JH, Park JW, Lee C, et al. ERG immunohistochemistry and clinicopathologic characteristics in Korean prostate adenocarcinoma patients. Korean J Pathol 2012; 46: 423-428.

19. Kimura T, Furusato B, Miki J, et al. Expression of ERG oncoprotein is associated with a less aggressive tumor phenotype in Japanese prostate cancer patients. Pathol Int 2012; 62: 742-748.

20. Wu F, Ding S, Lu J. Truncated ERG proteins affect the aggressiveness of prostate cancer. Med Hypotheses 2013; 80: 490-493.

21. Gasi Tandefelt D, Boormans JL, van der Korput HA, et al. A 36gene signature predicts clinical progression in a subgroup of ERGpositive. Eur Urol 2013; 10.1016/j.eururo.2013.02.039.

22. Shah RB. Clinical applications of novel ERG immunohistochemistry in prostate cancer diagnosis and management. Adv Anat Pathol 2013; 20: 117-124.

23. Yaskiv O, Zhang X, Simmerman K, et al. The utility of ERG/P63 double immunohistochemical staining in the diagnosis of limited cancer in prostate needle biopsies. Am J Surg Pathol 2011; 35: 1062-1068.

24. Tomlins SA, Palanisamy N, Siddiqui J, et al. Antibody-based detection of ERG rearrangements in prostate core biopsies, including diagnostically challenging cases: ERG staining in prostate core biopsies. Arch Pathol Lab Med 2012; 136: 935-946.

25. Shah RB, Tadros Y, Brummell B, et al. The diagnostic use of ERG in resolving an "atypical glands suspicious for cancer" di- agnosis in prostate biopsies beyond that provided by basal cell and alpha-methylacyl-CoA-racemase markers. Hum Pathol 2013; 44: 786-794.

\section{Address for correspondence}

\section{Krzysztof Okoń MD, PhD}

Katedra Patomorfologii CMUJ

ul. Grzegórzecka 16

31-531 Kraków

e-mail:k.okon@uj.edu.pl 